\title{
Design of a MIMO-OFDM Baseband Receiver for Next-Generation Wireless LAN
}

\author{
Zih-Yin Ding, Chi-Yun Chen and Tzi-Dar Chiueh \\ Graduate Institute of Electronics Engineering and Department of Electrical Engineering \\ National Taiwan University, Taipei, Taiwan, 10617
}

\begin{abstract}
In this paper, based on the IEEE 802.11n proposal [1], a MIMO-OFDM baseband receiver design for nextgeneration WLAN is proposed. A MIMO-OFDM receiver with algorithms for timing and frequency synchronization and MIMO detection is designed and simulated. Moreover, the circuits for all functional blocks in the receiver are also designed. Functional simulation results demonstrate that the proposed receiver design is capable of high link throughput with efficient spectrum utilization and is suitable for the application of next-generation wireless LAN.
\end{abstract}

\section{INTRODUCTION}

Due to the ability of coping with frequency selective fading and excellent spectral utilization efficiency, Orthogonal Frequency Division Multiplexing (OFDM) technology had been adopted in several standards, e.g. IEEE $802.11 \mathrm{a} / \mathrm{g}$ Wireless Local Area Network (WLAN) standard, IEEE 802.16 and Digital Video Broadcast-Terrestrial (DVB-T). In the nextgeneration WLAN standard, increasing the link throughput reliably to support a number of novel multi-media applications is a key point of standard development [2].

Recently, antenna array processing technology, also called Multiple Input Multiple Output (MIMO), has drawn lots of attention. It exploits multiple antennas at transmitter (TX) and/or receiver (RX) to enhance the transmission reliability via diversity gain and/or transmission data rate through multiplexing gain. When the MIMO technology with multiplexing gain is used, the spectral efficiency and capacity can be enhanced linearly with the antenna dimension [3]. Therefore, the combination of MIMO and OFDM, called MIMO-OFDM technology, is a promising candidate that has been applied in the next-generation WLAN to provide high link throughput and spectrum efficiency.

In this paper, we propose a MIMO-OFDM baseband receiver design compliant to the IEEE $802.11 \mathrm{n}$ proposal [1] with two transmit antennas and two receive antennas configuration. Algorithms for timing and frequency synchronization and MIMO signal detection are designed. In additional, the circuits of each functional block are designed and simulated. The functional simulation results of the proposed receiver establish the validity of its effectiveness in the high throughput WLAN application.

This paper is organized as follows. In Section II, a MIMOOFDM system model as well as its packet format are described. Section III depicts the receiver architecture. The algorithm and circuit design will also be introduced. In Section
IV, the simulation results are provided. Section V concludes this paper.

\section{System Model Description and Packet Format}

\section{A. System Model Description}

A $2 \times 2$ MIMO-OFDM system is illustrated in Fig. 1. The transmitted data bits after encoding are multiplexed into two spatial streams, interleaved and mapped to QAM symbols respectively. The transmitted symbol vector on frequency tone $k$ is $\mathbf{x}_{k}$ which is pre-processed via

$$
\mathbf{s}_{k}=\mathbf{Q}_{k} \cdot \mathbf{x}_{k}
$$

where $\mathbf{s}_{k}$ is the transmitted symbol vector after MIMO encoding and $\mathbf{Q}_{k}$ is the beamforming matrix. In the basic mode, transmitted signal is not beamformed and $\mathbf{Q}_{k}$ is simply a unity matrix. In the beamforming mode, $\mathbf{Q}_{k}$ is derived from the Singular Value Decomposition (SVD) of the channel matrix known by the transmitter. For example, if the MIMO channel matrix of the $k$ th frequency tone $\mathbf{H}_{k}$ can be decomposed using SVD as

$$
\mathbf{H}_{k}=\mathbf{U}_{k} \cdot \mathbf{D}_{k} \cdot \mathbf{V}_{k}^{H},
$$

where the $\mathbf{U}_{k}$ and $\mathbf{V}_{k}$ are unitary matrices and $\mathbf{D}_{k}$ is a diagonal matrix, then $\mathbf{V}_{k}$ matrix is set as $\mathbf{Q}_{k}$.

After the pre-processing, OFDM modulation including IFFT and Cyclic Prefix (CP) extension is applied. The RX removes CP and demodulates the received signal with FFT. The received symbol vector at the $k$ th frequency tone can be expressed as

$$
\mathbf{r}_{k}=\mathbf{H}_{k} \cdot \mathbf{s}_{k}+\mathbf{w}_{k}
$$

where $\mathbf{w}_{k}$ is an Additive White Gaussian Noise (AWGN) vector.

The received symbol vector $\mathbf{r}_{k}$ is decoded with the channel information which is derived from the orthogonal training sequence to separate the transmitted spatial streams. Each decoded stream is demapped, de-interleaved, and sent to the Viterbi decoder to find the most probable transmitted bit sequence.

\section{B. Packet Format}

The packet format used in the simulation is gleaned from [1] and shown in Fig. 2. The first three parts, including legacy short training field (L-STF), long training field (L-LTF) and signal field (L-SIG), are identical to those in the IEEE 802.11a packet to preserve backward compatibility. The following high 


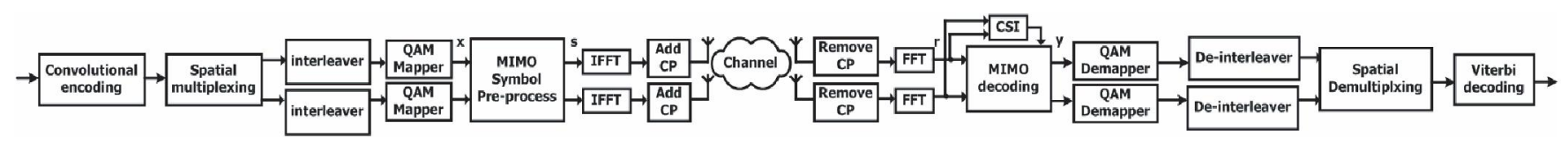

Fig. 1. Equivalent model of the MIMO-OFDM transceiver system

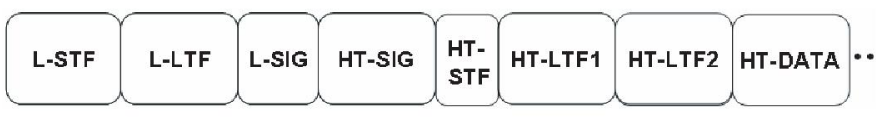

Fig. 2. Packet format

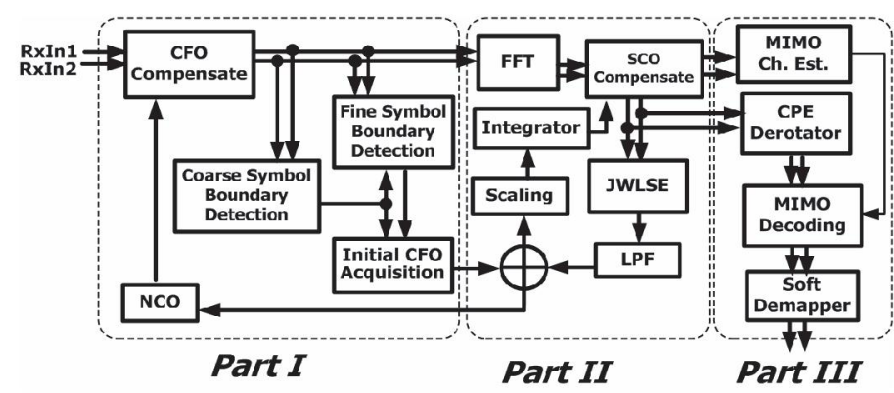

Fig. 3. Receiver architecture

throughput signal field (HT-SIG) and training fields (HTSTF, HT-LTF) are used for fine Automatic Gain Control (AGC) tuning and MIMO channel estimation, respectively. For estimation of the full MIMO channel, the number of high throughput long training field will be the same as the number of transmitted spatial streams. Finally, the high throughput data field (HT-DATA) contains the transmitted data payload of the spatial streams.

\section{ReCEIVER ARChiteCtURE AND FUnCtION INTRODUCTION}

The overall receiver architecture is illustrated in Fig. 3. For clarity, it is divided into three parts. Part I handles initial synchronization and time-domain carrier frequency offset (CFO) compensation. Part II is responsible for FFT operation and joint estimation of residual CFO and sampling clock offset (SCO). The $\mathrm{CFO} / \mathrm{SCO}$ estimation is filtered with a loop filter and accumulated to compensate $\mathrm{CFO} / \mathrm{SCO}$ in time/frequency domain, respectively. Channel estimation and data recovery are conducted in part III. The received frequency-domain symbol vector is decoded with VBLAST/SVD decoding algorithm in basic/beamforming mode. The decoded symbols are demapped with a soft demapper which determines bit metric for each bit. The bit metrics are sent to outer receiver to perform deinterleaving and soft Viterbi to decode the data bit sequence. In the following, the operations and circuit design of all functional blocks will be introduced.

\section{A. Initial Synchronization}

Initial synchronization includes packet detection, coarse symbol boundary detection, CFO estimation and fine symbol boundary detection.
1) Packet detection: The receiver detects the packet with normalized delay correlation of the received signal [4]. The normalized delay correlation $N(k)$ is given by

$$
N(k)=\frac{\sum_{n=k-L+1}^{k}\left(r(n) \cdot r^{*}(n-d)\right)}{\sum_{n=k-L+1}^{k}|r(n)|^{2}},
$$

where $r(n)$ is the combination of received signals at two antennas at time $n, k$ is time index, and $L$ is summation length. The delay $d$ is set as the period of short preamble. When the delay correlation value exceeds the threshold, the packet is detected.

2) Coarse symbol boundary detection: After AGC has settled, the receiver determines the coarse symbol boundary through the falling edge of the moving average of delay correlation.

3) CFO estimation: The phase of delay correlation corresponds to the CFO effect and is utilized in the estimation value of $\mathrm{CFO}$ by

$$
\Delta \hat{f}=\frac{\angle R\left(k_{o p t}\right)}{2 \pi \cdot d \cdot T_{S}},
$$

where $k_{\text {opt }}$ is the time index of symbol boundary and $T_{S}$ is sampling period. The $\mathrm{CFO}$ will be calculated again at long preamble to find a more accurate estimation. The hardware of coarse symbol boundary and $\mathrm{CFO}$ estimation is depicted in Fig. 4.

4) Fine symbol boundary detection: It is detected with a matched filter using long preamble. The output of matched filter is

$$
M(k)=\sum_{n=0}^{N-1} r(k-n) \cdot C(n),
$$

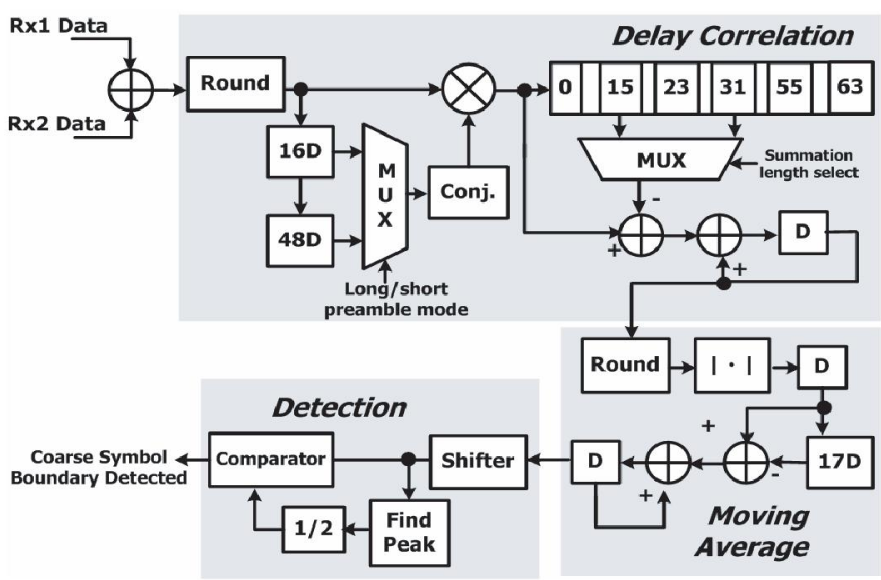

Fig. 4. Circuit design of coarse symbol boundary detection. 
where $N$ is the period of long preamble and $C(n)$ is the complex conjugate of L-LTF. For hardware reduction, $C(n)$ is quantized to 1 bit [5]. The matched filter output $M(k)$ characterizes the channel impulse response (CIR) and we can find a time-domain window that contains maximum energy of CIR through moving average of the matched filter output. The starting point of the window is determined as the symbol boundary to guarantee minimum inter-symbol interference (ISI). Moreover, matched filter output at this point must exceed a proportion of the maximum value of matched filter to avoid the variance of the boundary caused by channel noise.

\section{B. Demodulation}

The demodulation is performed via a 64-point FFT operation which is implemented with a radix $2 / 4 / 8$ architecture [6]. The multiplication with twiddle factors $W^{N / 8}, W^{3 N / 8}$, $W^{5 N / 8}$, and $W^{7 N / 8}$ can be replaced by shifters and adders to reduce hardware complexity [7]. Consequently, the number of complex multipliers is reduced to one.

\section{Tracking of Frequency Offset}

After CFO compensation, the residual CFO is relatively small. However, the accumulated rotation effect caused by the residual $\mathrm{CFO}$ and $\mathrm{SCO}$ can still deteriorate system performance and it needs additional compensations. The residual CFO can be estimated [8] by

$$
\delta \cong \frac{\sum_{k} w_{k} y_{k}}{K \cdot \sum_{k} w_{k}}
$$

where $y_{k}$ is the phase differences of the received pilots in two successive symbols and $w_{k}$ is the product of the two pilots' magnitude. The estimated $\mathrm{CFO}$ and SCO are compensated in time domain and frequency domain, respectively. The estimation result is passed through a loop filter to remove variance caused by noise and inter-carrier interference (ICI).

The hardware implementation employs a COrdinate and Rotation DIgital Computer (CORDIC) [9] to derive the phase and magnitude of the received pilots. Because the time interval of occurrence of pilots is larger than the latency of CORDIC and divider, the hardware can be shared for the calculation of all pilots.

\section{Channel Estimation and Data Recovery}

The receiver needs channel state information derived from channel estimation to decode the received frequency-domain symbols. These operations are done in part III.

1) Channel estimation: The MIMO channel estimation is evaluated from the recived HT-LTF. Because the HT-LTF is transmitted with tone interleaving technique [1], the transmitted training symbols in two transmit antennas are orthogonal to each other. When SNR is low, the channel estimation result is filtered for smoothing [10]. This is equivalent to the time domain CIR using a window filter to remove undesirable noise and improve the channel estimation quality.
2) MIMO signal decoding: The received symbol vector at each subcarrier must be decoded to separate transmitted symbols among different antennas. The detection method depends on the transmission mode.

a) Basic mode: In this mode, the receiver uses VBLAST algorithm [11] to decode the MIMO signal. Therefore, the decoding vector which is calculated from the pseudo-inverse of the deflated channel matrix and the decoding order must be determined in each iteration.

b) Beamforming mode: In this mode, the received symbol vector is

$$
\mathbf{r}_{k}=\mathbf{H}_{k} \cdot \mathbf{s}_{k}=\left(\mathbf{U}_{k} \cdot \mathbf{D}_{k}\right) \cdot \mathbf{x}_{k}=\mathbf{M}_{k} \cdot \mathbf{x}_{k} .
$$

Because $\mathbf{U}_{k}$ is a unitary matrix and $\mathbf{D}_{k}$ is a diagonal matrix, the decoding matrix can be represented as

$$
\mathbf{M}_{k}^{-1}=\mathbf{D}_{k}^{-1} \cdot \mathbf{U}_{k}^{H}=\mathbf{D}_{k}^{-2} \cdot \mathbf{M}_{k}^{H} .
$$

The matrix $\mathbf{M}_{k}$ can be estimated from beamformed HT-LTF. Since $\mathbf{D}_{k}$ is a real diagonal matrix, the calculation of decoding matrix is simpler than that in the basic mode. In addition, the Ordered SUccessive Cancellation (OSUC) concept which is originated in VBLAST is also applied to enhance the decoding performance.

3) Soft demapping: The soft demapper calculates Log Likelihood Ratio $(L L R)$ as bit metric for each bit which can be the input of the soft Viterbi decoder. In SISO case, the bit metric of the $m$ th bit of the symbol transmitted on $k$ th frequency tone, can be calculated as

$$
\begin{aligned}
\operatorname{LLR}\left(b_{k, m}\right) & =\log \frac{\operatorname{Pr}\left(b_{k, m}=1 \mid r_{k}\right)}{\operatorname{Pr}\left(b_{k, m}=0 \mid r_{k}\right)} \\
& \approx C S I_{k} \cdot\left(\min _{\hat{x} \in S_{0}^{m}}\left(y_{k}-\hat{x}\right)-\min _{\hat{x} \in S_{1}^{m}}\left(y_{k}-\hat{x}\right)\right),
\end{aligned}
$$

where $S_{j}^{m}$ is the set of all constellation points that has $m$ th bit equal to $j, y_{k}$ are the received and equalized symbol on $k$ th frequency tone and $C S I_{k}$ is the post-detection SNR on $k$ th frequency tone. Due to the symmetry of Gray coding, the second term on the right of (10) can be simplified with linear piecewise functions [5]. In MIMO case, the LLR can also be approximated with (10) while the $C S I_{k}$ is the Signal to Interference and Noise Ratio (SINR) in each transmitted spatial stream after detection [12].

\section{Simulation Result}

The functional simulation is under TGn Channel Model D which is a typical official channel model and has delay spread equal to $50 \mathrm{~ns}$. The center frequency is set to $5320 \mathrm{MHz}$. The CFO and SCO are both $-13.7 \mathrm{ppm}$ according to the TGn comparison criteria [13]. The OFDM parameters used in the simulation are shown in Table I. It is demonstrated in Fig. 5 that the SVD decoding with OSUC can improve the performance by about $10 \mathrm{~dB}$ as compared to the original SVD decoding under PER $=10 \%$. In addition, the performance of the SVD decoding with OSUC is better than the VBLAST decoding and its complexity in the receiver is lower than the VBLAST decoding. Fig. 6 validates the effect of soft 
demapping combined with soft Viterbi decoding. When the data rate is $108 \mathrm{Mbps}$, the SNR improvement of soft-decision Viterbi decoding is about $8 \mathrm{~dB}$ over hard-decision Viterbi decoding.

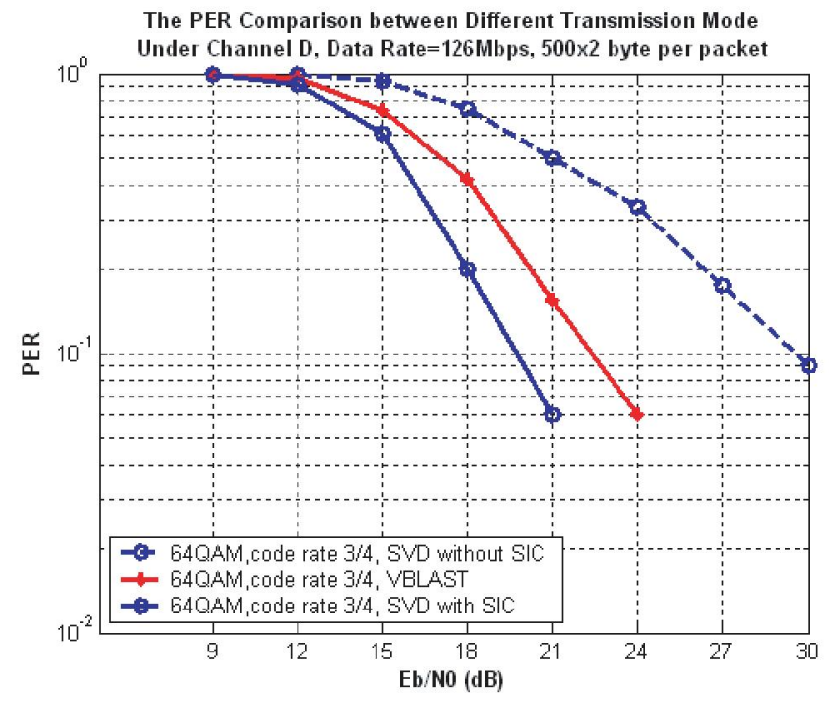

Fig. 5. PER comparison between basic and beamforming modes.

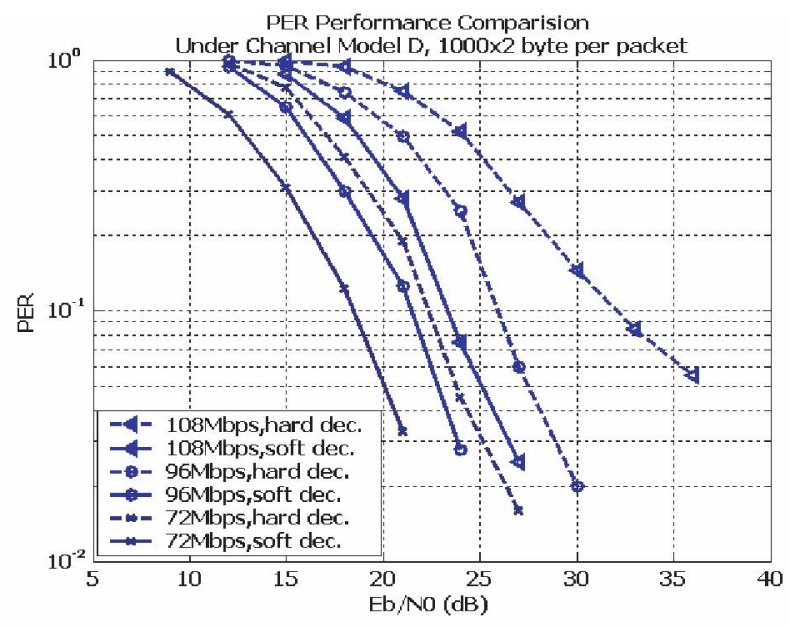

Fig. 6. PER performance under difference data rates.

\section{CONClusion}

In this paper, a MIMO-OFDM receiver design for nextgeneration WLAN application is presented. The algorithms and circuits for timing and frequency synchronization, tracking of frequency offset, MIMO signal detection and soft demapper in MIMO system are designed and simulated. The functional simulation results have demonstrated the performance enhancement and applicability of the proposed receiver design in the next-generation high throughput WLAN.
TABLE I

\begin{tabular}{|c|c|}
\hline \multicolumn{2}{|c|}{ OFDM Parameters } \\
\hline \hline \multicolumn{2}{|c|}{} \\
FFT size & 64 \\
Subcarrier in use & 52 \\
Subcarrier spacing & $312.5 \mathrm{kHz}$ \\
FFT period & $3.2 \mathrm{us}$ \\
Guard interval duration & $0.8 \mathrm{us}$ \\
OFDM duration & $4.0 \mathrm{us}$ \\
\hline
\end{tabular}

\section{ACKNOWLEDGMENT}

This work is supported in part by NSC, Taiwan under Grant no. NSC94-2218-E-002-059 and NSC94-2219-E-002-004.

\section{REFERENCES}

[1] TGn Sync website: http://www.tgnsync.org

[2] IEEE 80211 TGn, "Functional Requirements," DCN:11-03-0813-r9.

[3] G. J. Foschini and M. J. Gans, "On the limits of wireless communications in a fading environment when using multiple antennas," Wireless Personal Communications, vol. 6, pp. 311-335, Mar. 1998

[4] T. M. Schmidl and D. C. Cox, "Robust frequency and timing synchronization for OFDM," IEEE Transactions on Communications, Vol.45, Issue 12, pp. 1613-1621, Dec. 1997

[5] Richard van Nee and Ramjee Prasad, "OFDM for Wireless Multimedia. Communications", London, UK: Artech House, 2000.

[6] Lihong Jia, Yonghong Gao, Jouni Isoaho and Hannu Tenhunen, "A New VLSI-Oriented FFT Algorithm and Implementation," Proc. of the IEEE 11th Annual International ASIC Conference, pp. 337-341, Sep. 13-16, 1998

[7] Yeong-Terng Lin, "Design and implementation of a Variable-Length FFT Processor for OFDM Systems," Master thesis, Dept of Electrical Engineering National Taiwan University, Taipei, Taiwan, Jun. 2001.

[8] Pei-Yun Tsai, Hsin-Yu Kang, and Tzi-Dar Chiueh,'Joint weighted least squares estimation of frequency and timing offset for OFDM systems over fading channels," IEEE Vehicular Technology Conference, vol. 4, pp. 2543-2547, Apr. 2003

[9] Ray Andraka, "A survey of CORDIC algorithms for FPGAs," Proceedings of the 1998 ACM/SIGDA sixth international symposium on Field programmable gate arrays, pp. 191-200, Feb. 22-24, 1998, Monterey, CA.

[10] Pei-Yun Tsai and Tzi-Dar Chiueh, "Frequencydomain interpolationbased channel estimation in pilot-aided OFDM systems," Proc. IEEE Vehicular Technology Conference, Vol. 1, pp. 420-424, May 2004

[11] P.W. Wolniansky, G.J. Foschini, G.D. Golden, and R.A. Valenzuela, "VBLAST: an architecture for realizing very high data rates over the richscattering wireless channel," URSI International Symposium on Signals, Systems, and Electronics, pp. 295-300, Sept. 29- Oct. 2, 1998

[12] Kee-Bong Song and Syed Aon Mujtaba, "A Low Complexity SpaceFrequency BICM MIMO-OFDM System for Next Generation WLANs," Proc. of IEEE GLOBECOMM, Vol.2, pp.1059-1063, Dec. 2003

[13] IEEE 802.11 TGn, "Comparison criteria," DCN: 11-03-0814-rl6. 